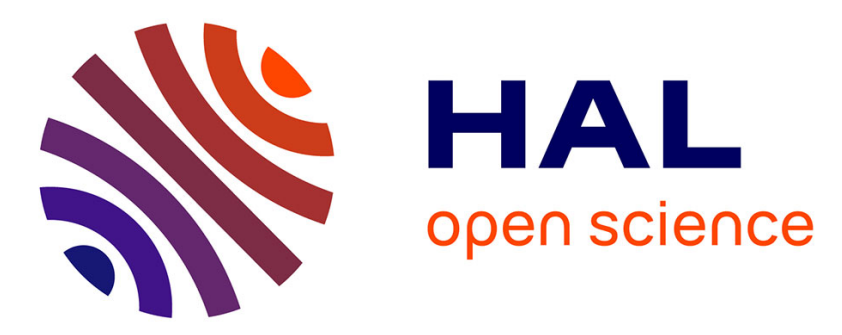

\title{
Influence de la température sur le taux de pulvérisation de cibles de silicium et germanium
}

\author{
J. Nizam, N. Benazeth-Colombie
}

\section{To cite this version:}

J. Nizam, N. Benazeth-Colombie. Influence de la température sur le taux de pulvérisation de cibles de silicium et germanium. Revue de Physique Appliquée, 1975, 10 (4), pp.183-186. 10.1051/rphysap:01975001004018300 . jpa-00243903

\section{HAL Id: jpa-00243903 https://hal.science/jpa-00243903}

Submitted on 1 Jan 1975

HAL is a multi-disciplinary open access archive for the deposit and dissemination of scientific research documents, whether they are published or not. The documents may come from teaching and research institutions in France or abroad, or from public or private research centers.
L'archive ouverte pluridisciplinaire HAL, est destinée au dépôt et à la diffusion de documents scientifiques de niveau recherche, publiés ou non, émanant des établissements d'enseignement et de recherche français ou étrangers, des laboratoires publics ou privés. 


\title{
REVUE DE PHYSIQUE APPLIQUÉE
}

Supplément au * Journal de Physique*

Classification

Physics Abstracts

7.180

\section{INFLUENCE DE LA TEMPÉRATURE SUR LE TAUX DE PULVÉRISATION DE CIBLES DE SILICIUM ET GERMANIUM}

\author{
J. NIZAM et N. BENAZETH-COLOMBIE \\ Laboratoire de Physique des Solides (*), \\ Université Paul-Sabatier, 118, route de Narbonne, 31077 Toulouse Cedex, France. \\ (Reçu le 13 mai 1974, révisé le 13 janvier 1975, accepté le 18 février 1975)
}

\begin{abstract}
Résumé. - Nous avons étudié, en fonction de la température $\left(50-500{ }^{\circ} \mathrm{C}\right)$ la variation du taux de pulvérisation $S$ de cibles monocristallines de $\mathrm{Si}$ et de Ge, bombardées par de fortes doses ( $>10^{17}$ ions $/ \mathrm{cm}^{2}$ ) d'ions $\mathrm{Ar}^{+}$ayant une énergie de $40 \mathrm{keV}$.

Ces mesures de $S$ ont permis de déterminer les conditions de température des cibles dans lesquelles on ne perturbe pas la structure du réseau cristallin $\left(\sim 350^{\circ} \mathrm{C}\right.$ pour le $\mathrm{Ge}$ et $\gtrsim 400^{\circ} \mathrm{C}$ pour le $\mathrm{Si}$ ).

Nous avons déterminé également les taux de pulvérisation caractéristiques des monocristaux parfaits de Ge.
\end{abstract}

\footnotetext{
Abstract. - Si and Ge single crystal targets were bombarded with high doses of $40 \mathrm{keV} \mathrm{Ar}+$ ions ( $>10^{17}$ ions $/ \mathrm{cm}^{2}$ ). The variation of the sputtering yield $S$ as function of the target temperature $\left(50-500{ }^{\circ} \mathrm{C}\right)$ has been studied.

The measurements of $S$ lead to a determination of the annealing temperature $\left(\sim 350^{\circ} \mathrm{C}\right.$ for $\mathrm{Ge}$ and $\gtrsim 400^{\circ} \mathrm{C}$ for $\mathrm{Si}$ ).

The sputtering yields of perfect single-crystal Ge targets have been measured.
}

1. Introduction. - L'implantation ionique est maintenant une technique couramment utilisée pour le dopage des semiconducteurs. Mais le bombardement ionique crée dans le matériau dopé des défauts qui modifient sa structure et ses propriétés électriques. Plusieurs méthodes d'investigation ont été utilisées pour déterminer les conditions de guérison des défauts, telles que la diffusion de Rutherford [1], l'effet Hall [2], la microscopie électronique [3] ainsi que la pulvérisation cathodique [4]. Le taux de pulvérisation et la distribution spatiale des atomes éjectés à partir de monocristaux sont en effet liés à l'ordre du réseau cristallin en volume et en surface.

Nous avons étudié précédemment l'évolution, en fonction de la température, des diagrammes des atomes

(*) Laboratoire associé au C. N. R.S. éjectés à partir de monocristaux de Si et de Ge [5]. Nous présentons ici les résultats obtenus concernant la variation du taux de pulvérisation $S$ de cibles monocristallines de $\mathrm{Si}$ et de $\mathrm{Ge}$ bombardées par de fortes doses $\left(>10^{17}\right.$ ions $/ \mathrm{cm}^{2}$ ) d'ions $\mathrm{Ar}^{+}$de $40 \mathrm{keV}$. Cette étude nous a permis de déterminer les conditions de température dans lesquelles le réseau cristallin est restauré.

2. Dispositif expérimental. - Un accélérateur d'ions positifs permet d'obtenir des ions $\mathrm{Ar}^{+}$ayant une énergie de $40 \mathrm{keV}$. La densité du faisceau ionique au niveau de la cible est de l'ordre de $30 \mu \mathrm{A} / \mathrm{mm}^{2}$. Un four permet de chauffer la cible jusqu'à une température de $550^{\circ} \mathrm{C}$. Cette température est déterminée avec une incertitude de $\pm 2^{\circ} \mathrm{C}$, à l'aide d'un thermocouple chromel-alumel.

La cible est placée dans une enceinte où la pression 
est de l'ordre de $10^{-7}$ torr. Les atomes éjectés sont recueillis sur une plaque de verre placée à $15 \mathrm{~mm}$ audessus de la surface de la cible.

Les échantillons étudiés sont des monocristaux de $\mathrm{Ge}$ et de Si orientés de telle sorte que leur surface soit parallèle respectivement aux plans (111), (100), (110).

Le taux de pulvérisation est défini par $S=N_{\mathrm{a}} / N_{\mathrm{i}}$, où $N_{\mathrm{a}}$ est le nombre d'atomes arrachés et $N_{\mathrm{i}}$ est le nombre d'ions incidents.

$S$ est déterminé par la relation :

$$
S=\frac{\Delta_{m}}{M_{2}} N_{0} \frac{e}{I t}
$$

où $\Delta_{m}$ est la perte de masse de la cible au cours du bombardement, $N_{0}$ est le nombre d'Avogadro, $M_{2}$ la masse atomique de la cible, $t$ la durée du bombardement et $I$ l'intensité du courant ionique incident. $\Delta_{m}$ est mesurée avec une incertitude de 0,005 mg. L'enregistrement du courant $I$ et l'intégration sur le temps permettent de déterminer la quantité $I t$. Le taux $S$ est alors déterminé avec une précision de $5 \%$.

3. Résultats expérimentaux. - 3.1 CiBles MONOCRISTALlinES. - Les figures 1, 2, 3, 4, 5 et 6 donnent les résultats expérimentaux concernant la variation du taux de pulvérisation de cibles monocristallines de Si et de Ge en fonction de la température de l'échantillon.

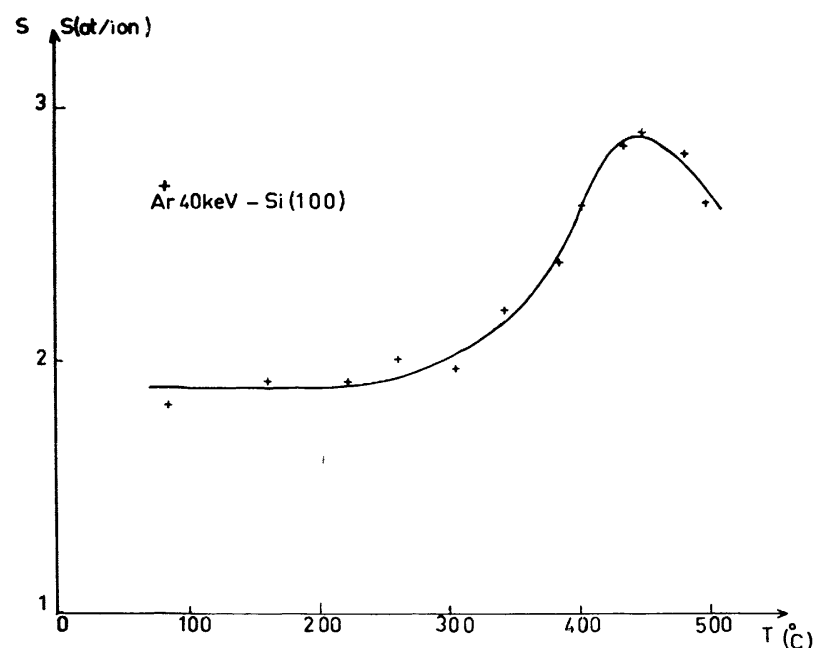

Fig. 1. - Variation de $S$ en fonction de la température pour $\mathrm{Si}$ (100).

Les courbes présentées dans ces figures présentent des caractéristiques dépendant de la nature et de l'orientation cristalline de la face bombardée. Ces caractéristiques sont :

— un palier du taux de pulvérisation $S$ de valeur $S_{\text {a }}$ pour les faibles températures et jusqu'à une température $T_{\mathrm{a}}$;

- une augmentation du taux $S$ dans un domaine de température $T_{\mathrm{a}}-T_{\mathrm{M}}$. Le maximum du taux $S_{\mathrm{M}}$ est atteint à la température $T_{\mathrm{M}}$;

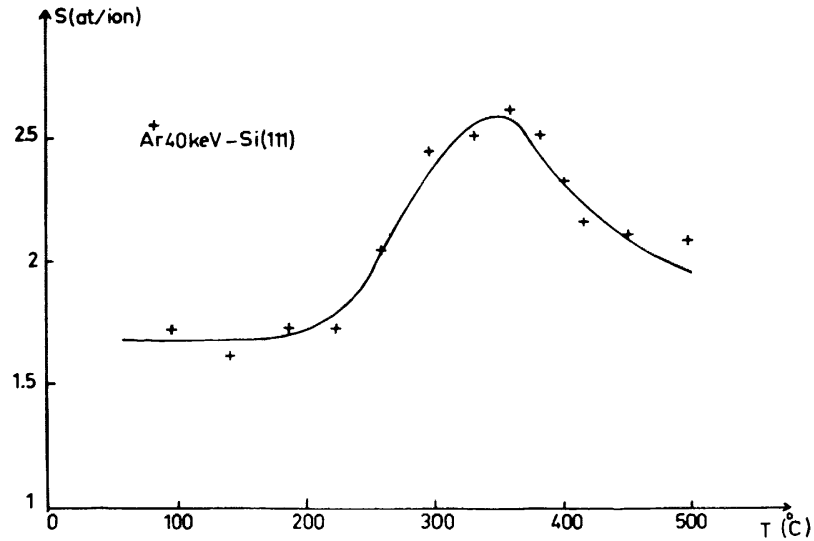

Fig. 2. - Variation de $S$ en fonction de la température pour Si (111).

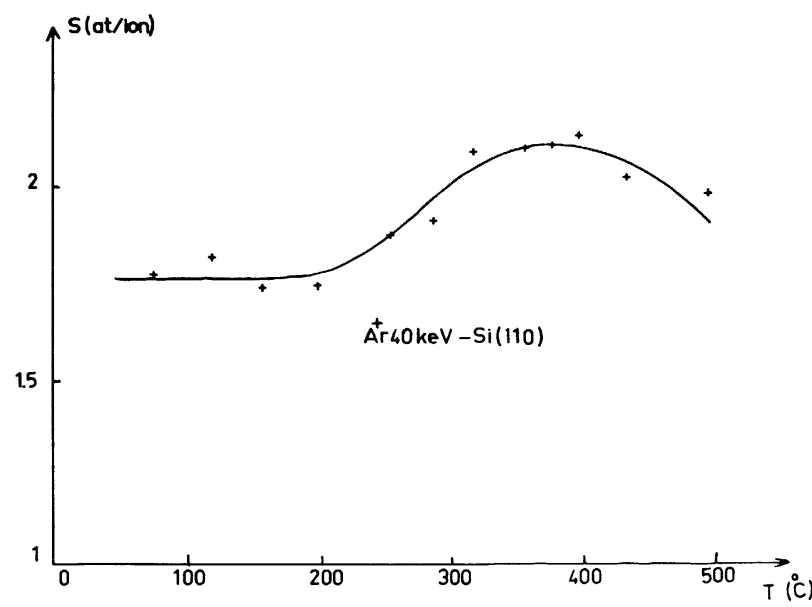

FIG. 3. - Variation de $S$ en fonction de la température pour $\mathrm{Si}$ (110).

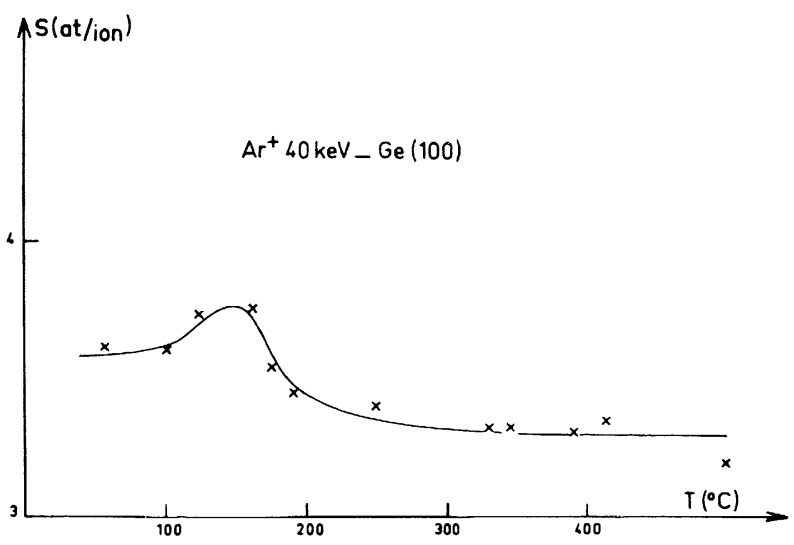

FIG. 4. - Variation de $S$ en fonction de la température pour $\mathrm{Ge}(100)$.

- une décroissance du taux $S$ pour des températures supérieures à $T_{\mathrm{M}}$. Dans le cas du germanium, cette décroissance est observée jusqu'à une température $T_{\mathrm{c}}$ à partir de laquelle le taux $S$ atteint un palier de valeur $S_{\mathrm{c}}$. Notons que dans nos expériences, ce palier n'est pas observé dans le cas du Si. 


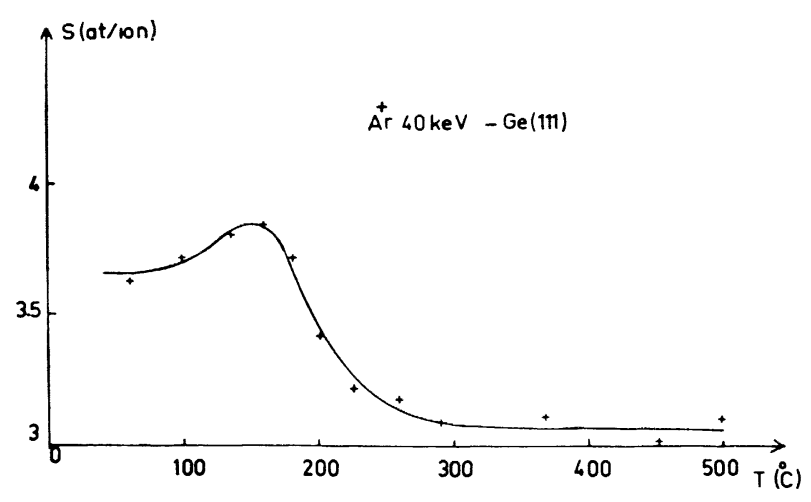

Fig. 5. - Variation de $S$ en fonction de la température pour Ge (111).

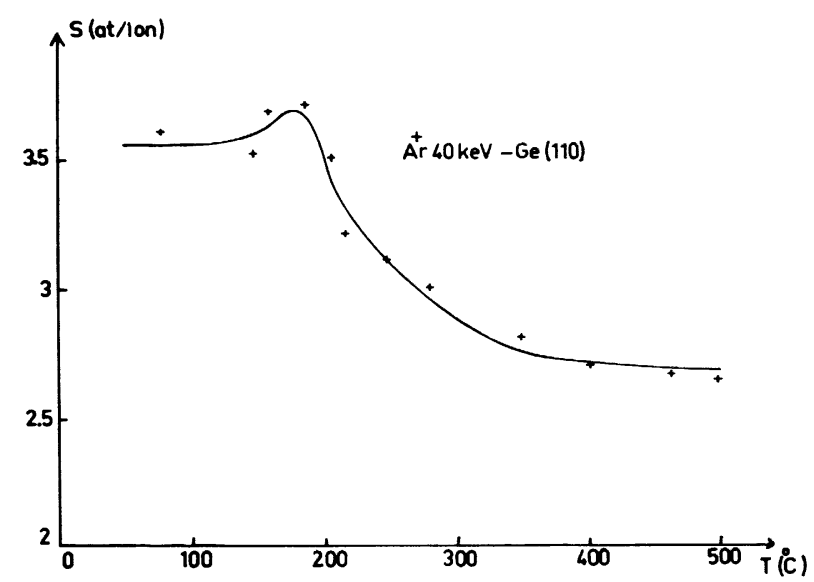

FIG. 6. - Variation de $S$ en fonction de la température pour $\mathrm{Ge}(110)$.

Les valeurs de $T_{\mathrm{a}}, T_{\mathrm{M}}, S_{\mathrm{a}}$ et $S_{\mathrm{M}}$ relatives aux différentes cibles du silicium sont résumées dans le tableau I.

Le tableau II donne les valeurs de $T_{\mathrm{a}}, T_{\mathrm{M}}, T_{\mathrm{c}}, S_{\mathrm{a}}, S_{\mathrm{M}}$ et $S_{\mathrm{c}}$ correspondant aux cibles de germanium.

\section{TABLEAU I}

\begin{tabular}{|c|c|c|c|c|}
\hline Cible & $\begin{array}{l}T_{\mathrm{a}} \\
{ }^{\circ} \mathrm{C}\end{array}$ & $\begin{array}{l}T_{\mathrm{M}} \\
{ }^{\circ} \mathrm{C}\end{array}$ & $S_{\mathrm{a}}$ & $S_{\mathrm{M}}$ \\
\hline $\mathrm{Si}(100)$ & 210 & 440 & 1,9 & 2,9 \\
\hline $\mathrm{Si}(111)$ & 190 & 350 & 1,7 & 2,6 \\
\hline $\mathrm{Si}(110)$ & 180 & 380 & 1,7 & 2,1 \\
\hline
\end{tabular}

\section{TABLEAU II}

\begin{tabular}{|c|c|c|c|c|c|c|}
\hline Cible & $\begin{array}{l}T_{\mathrm{a}} \\
{ }^{\circ} \mathrm{C}\end{array}$ & $\begin{array}{l}T_{\mathrm{M}} \\
{ }^{\circ} \mathrm{C}\end{array}$ & $\begin{array}{l}T_{\mathrm{c}} \\
{ }^{\circ} \mathrm{C}\end{array}$ & $S_{\mathrm{a}}$ & $S_{\mathrm{M}}$ & $S_{\mathrm{c}}$ \\
\hline $\mathrm{Ge}(100)$ & 100 & 150 & 350 & 3.6 & 3.75 & 3,3 \\
\hline Ge (111) & 80 & 150 & 300 & 3,6 & 3,85 & 3,05 \\
\hline $\mathrm{Ge}(110)$ & 120 & 180 & 380 & 3,6 & 3,7 & 2,7 \\
\hline
\end{tabular}

3.2 Discussion. - Nous avons interprété ces résultats en tenant compte d'une part de l'évolution, en fonction de la température, de la structure du réseau cristal- lin soumis au bombardement ionique et d'autre part des mécanismes d'éjection des atomes.

3.2.1 Cas du silicium. - Les paliers de taux de pulvérisation $S_{\mathrm{a}}\left[S_{\mathrm{a}}=1,9\right.$ pour la face $(100)$ et 1,7 pour les faces (111) et (110)] observés dans le domaine de température $50-200{ }^{\circ} \mathrm{C}$ s'interprètent comme étant dus à un état amorphe de la couche superficielle des cibles. En effet, comme nous l'avons montré dans un précédent article [5], les diagrammes d'éjection obtenus dans ce domaine de température sont isotropes et identiques à ceux obtenus à partir de cibles polycristallines, de plus, la valeur de $S_{\mathrm{a}}=1,9$ coïncide avec la valeur du taux $S$ mesurée pour un polycristal. $\mathrm{La}$ valeur légèrement plus faible obtenue sur $\mathrm{Si}(110)$ et $\mathrm{Si}$ (111) peut être attribuée à la transparence du substrat qui facilite la canalisation d'une partie des ions incidents malgré la perturbation de la couche superficielle.

Deux facteurs essentiels peuvent interpréter l'augmentation du taux de pulvérisation dans l'intervalle de température $T_{\mathrm{a}}-T_{\mathrm{M}}\left(200-400{ }^{\circ} \mathrm{C}\right)$. D'une part, les diagrammes d'éjection obtenus dans ce domaine sont anisotropes [5] et caractérisent une surface réarrangée dont la barrière de potentiel $E_{\mathrm{b}}$ est plus faible que celle d'un polycristal [7] ; cette diminution de $E_{\mathrm{b}}$ contribue à l'augmentation du taux $S$, celui-ci étant fonction de $E_{\mathrm{b}}^{-1}$ [8]. D'autre part, certains auteurs [9] ont montré que la pulvérisation thermique due aux thermal spikes se manifeste dans le cas du silicium polycristallin dans l'intervalle de température $200-400{ }^{\circ} \mathrm{C}$ et tend à augmenter le taux $S$. Ce processus pourrait, en partie, être responsable de la croissance de $S$ observée sur nos courbes expérimentales. Cependant, pour un monocristal, au fur et à mesure que la température du réseau augmente les défauts en volume se guérissent, la transparence du réseau augmente et la section efficace de collision diminue. Au-delà de $T_{\mathrm{M}}\left(\sim 400^{\circ} \mathrm{C}\right)$, les effets dus à la canalisation des particules incidentes sont prépondérants et on observe une décroissance de taux $S$ correspondant à une guérison progressive en volume du réseau cristallin. Ceci est en accord avec les résultats de Hafemeister et coll. [10], Eriksson et coll. [11] et de Hart et coll. [12].

3.2.2 Cas du germanium. - Le palier $S_{\mathrm{a}}=3,6$ (Fig. 4, 5 et 6) obtenu pour les différentes cibles de germanium et pour des températures inférieures à $T_{\mathrm{a}} \approx 100^{\circ} \mathrm{C}$ correspond à une couche superficielle amorphe de la cible. En effet, d'une part les diagrammes d'éjection obtenus dans ce domaine de température sont isotropes [5] et d'autre part, la valeur $S_{\mathrm{a}}=3,6$ coïncide avec la valeur théorique du taux $S$ donnée par Sigmund [8] pour un polycristal de germanium.

Au-delà de $T_{\mathrm{a}}$ le taux de pulvérisation augmente légèrement de $5 \%$ environ, alors que dans le cas du silicium nous avons observé une augmentation du taux $S$ de l'ordre de $40 \%$. On pourrait attribuer ce comportement différent au fait que, dans le cas du germanium, l'effet des thermal spikes n'intervient qu'à 
une température supérieure à $500^{\circ} \mathrm{C}$ [9] et par conséquent l'augmentation de $S$ entre 100 et $170{ }^{\circ} \mathrm{C}$ serait due uniquement à la diminution de la barrière de potentiel $E_{\mathrm{b}}$ lors du réarrangement de la surface [7]. Dans l'intervalle de température $T_{\mathrm{M}}-T_{\mathrm{c}}\left(150-350^{\circ} \mathrm{C}\right)$, les défauts se guérissent, la transparence augmente et le taux $S$ diminue.

Au-delà de $T_{\mathrm{c}}\left(\sim 350^{\circ} \mathrm{C}\right)$ le taux $S$ reste constant et égal à $S_{\mathrm{c}}$. $T_{\mathrm{c}}$ peut être considéré comme la température de guérison totale des défauts et les valeurs $S_{\mathrm{c}}$ obtenues pour les différentes faces sont caractéristiques du monocristal considéré (Tableau II).

La température de guérison totale des défauts $T_{\mathrm{c}} \sim 350^{\circ} \mathrm{C}$ est en accord avec les résultats de Holmen et coll. [13] et de Parsons [14].
4. Conclusion. - La pulvérisation cathodique semble une bonne méthode pour étudier la guérison des défauts créés en surface et en volume, lors du bombardement ionique de cibles semiconductrices.

Etant donné que dans le cas du germanium la pulvérisation thermique apparaît pour des températures supérieures à $500^{\circ} \mathrm{C}$, il serait nécessaire d'étendre le domaine de variation de la température $T$ jusqu'à environ $700^{\circ} \mathrm{C}$. La comparaison des courbes de variation du taux de pulvérisation en fonction de $T$ obtenues avec le silicium et le germanium permettrait alors de mettre en évidence le rôle des thermal spikes et vérifier ainsi l'hypothèse que nous proposons pour interpréter les résultats obtenus avec le silicium.

\section{Bibliographie}

[1] Mayer, J. W., Eriksson, L., Picraux, S. T. et Davies, J. A., Can. J. Phys. 46 (1968) 663.

[2] Pavlov, P. V., Zorin, E. I., Tetel'baum, D. I. et Popov, Yu. S., Sov. Phys. Solid State 6 (1965) 2577.

[3] Mazey, D. J., Nelson, R. S. et Barnes, R. S., Phil. Mag. 17 (1968) 1145.

[4] Zwangobani, E. et Mac Donal, R. J., Phys. Lett. 32A (1970) 308.

[5] Nizam, J. et Benazeth-Colombie, N., C. R. Hebd. Séan. Acad. Sci. 278B (1974) 1095.

[6] Seitz, F. et Koenler, J. S., Progress in Solid State Physics (Academic Press Inc., N.-Y.) 1957, 30.
[7] Lander, J. J. et Morrison, J., J. Appl. Phys. 34 (1963) 1403.

[8] Sigmund, P., Phys. Rev. 184 (1969) 383.

[9] Nelson, R. S., Phil. Mag. 11 (1965) 291.

[10] Hafemeister, D. W. et De-WaArd, D. H., Phys. Rev. 137 (1973) 3014.

[11] Eriksson, L., Davies, J. A., Johansson, N. G. E. et Mayer, J. W., J. Appl. Phys. 40 (1969) 842.

[12] Hart, R. R. et Marsh, O. J., Appl. Phys. Lett. 14 (1969) 2225.

[13] Holmen, G. et Hogberg, P., Rad. Effects 12 (1972) 77.

[14] Parsons, J. R., Phil. Mag. 12 (1965) 1159. 